\title{
A Note on Legendre-Fenchel Conjugate of the Product of Two Positive-Definite Quadratic Forms
}

\author{
Yong Xia
}

Received: 25 April 2013 / Revised: 14 June 2013 / Accepted: 20 June 2013 /

Published online: 24 July 2013

(C) Operations Research Society of China, Periodicals Agency of Shanghai University, and

Springer-Verlag Berlin Heidelberg 2013

\begin{abstract}
The Legendre-Fenchel conjugate of the product of two positive-definite quadratic forms was posted as an open question in the field of nonlinear analysis and optimization by Hiriart-Urruty ['Question 11' in SIAM Review 49, 255-273, 2007]. Under a convexity assumption on the function, it was answered by Zhao [SIAM J. Matrix Analysis \& Applications 31(4), 1792-1811, 2010]. In this note, we answer the open question without making the convexity assumption.
\end{abstract}

Keywords Legendre-Fenchel conjugate · Quadratic form

\section{Introduction}

The Legendre-Fenchel conjugate of the function $h(y)$ is defined as

$$
h^{*}(x)=\sup _{y \in R^{n}} x^{\mathrm{T}} y-h(y)
$$

For example, if $h(y)=\frac{1}{2} y^{\mathrm{T}} A y$, where $A$ is positive definite, then $h^{*}(x)=\frac{1}{2} x^{\mathrm{T}} A^{-1} x$. In the field of nonlinear analysis and optimization, J.B. Hiriart-Urruty [3] raised an open question: What is the expression or formula of the conjugate of the product

This research was supported by National Natural Science Foundation of China (Nos. 11001006 and 91130019/A011702), by the Fundamental Research Funds for the Central Universities (No. YWF-13-A01), and by the Fund of State Key Laboratory of Software Development Environment (No. SKLSDE-2013ZX-13).

\section{Y. Xia $(\bowtie)$}

State Key Laboratory of Software Development Environment, Key Laboratory of Mathematics, Informatics and Behavioral Semantics of the Ministry of Education, School of Mathematics and System Sciences, Beihang University, Beijing 100191, P.R. China

e-mail: dearyxia@gmail.com 
function

$$
f(y)=\frac{1}{4}\left(y^{\mathrm{T}} A y\right)\left(y^{\mathrm{T}} B y\right),
$$

where $A, B$ are two $n \times n$ positive-definite matrices.

Recently, Zhao [5] answered the open question under the assumption that $f(y)$ is convex. The main result is as follows.

Theorem 1.1 ([5]) Let the function $f(y)$ be convex. At any point $x \in \mathbb{R}^{n}$, the value of the conjugate $f^{*}(x)$ is finite, and $f^{*}(x)=0$ if $x=0$, otherwise if $x \neq 0$,

$$
f^{*}(x)=p(\alpha):=3 \alpha^{1 / 3}\left(\frac{x^{\mathrm{T}}(A+\alpha B)^{-1} x}{4}\right)^{2 / 3}
$$

where $\alpha$ is any real root of the univariate equation

$$
g(\alpha):=\alpha-\frac{x^{\mathrm{T}}(A+\alpha B)^{-1} A(A+\alpha B)^{-1} x}{x^{\mathrm{T}}(A+\alpha B)^{-1} B(A+\alpha B)^{-1} x}=0 .
$$

The following gives a sufficient condition under which the real root to Eq. (1) is unique.

Theorem 1.2 ([5]) If $\max \{\kappa(A), \kappa(B)\} \leqslant 2.5$, where $\kappa(\cdot)$ denotes the condition number, then $f(y)$ is convex and for any $x \neq 0$, there exists a unique real root to Eq. (1).

In this note, we answer the open question without the convexity assumption. Compared with the proof of Theorem 1.1 given in [5], our proof is simple and easy to understand. As an application, the sufficient condition (Theorem 1.2) is improved.

\section{Main Result}

In this section, we study the conjugate $f^{*}(x)=\sup _{y \in R^{n}} x^{\mathrm{T}} y-f(y)$ without assuming that $f(y)$ is convex.

Lemma 2.1 The conjugate $f^{*}(x)$ is finite. $f^{*}(x)=0$ if $x=0$, otherwise if $x \neq 0$, $f^{*}(x)>0$.

Proof The finiteness of $f^{*}(x)$ follows from

$$
\lim _{\|y\|_{2} \rightarrow \infty} x^{\mathrm{T}} y-\frac{1}{4}\left(y^{\mathrm{T}} A y\right)\left(y^{\mathrm{T}} B y\right) \leqslant \lim _{\|y\|_{2} \rightarrow \infty} x^{\mathrm{T}} y-\frac{\lambda_{\min }(A) \lambda \min (B)}{4}\|y\|_{2}^{4}=-\infty,
$$

where $\lambda_{\min }(A)>0$ and $\lambda_{\min }(B)>0$ are the minimal eigenvalues of $A$ and $B$, respectively. If $x=0$, it is trivial to verify $f^{*}(x)=0$. Now we assume $x \neq 0$. Since

$\max _{\epsilon}\left\{x^{\mathrm{T}}(\epsilon x)-f(\epsilon x)\right\}=\max _{\epsilon}\left\{x^{\mathrm{T}} x \epsilon-\frac{\left(x^{\mathrm{T}} A x\right)\left(x^{\mathrm{T}} B x\right)}{4} \epsilon^{2}\right\}=\frac{\left(x^{\mathrm{T}} x\right)^{2}}{\left(x^{\mathrm{T}} A x\right)\left(x^{\mathrm{T}} B x\right)}>0$,

we have $f^{*}(x)>0$. The proof is complete. 
Lemma 2.2 Suppose $x \neq 0 . y$ is a stationary point of $x^{\mathrm{T}} y-f(y)$ if and only if

$$
x^{\mathrm{T}} y-f(y)=p(\alpha),
$$

where $\alpha$ is a real root of the univariate equation (1).

Proof Suppose $y$ is a stationary point of $x^{\mathrm{T}} y-f(y)$. We have

$$
x=\nabla f(y)=\left(\frac{1}{2}\left(y^{\mathrm{T}} A y\right) B+\frac{1}{2}\left(y^{\mathrm{T}} B y\right) A\right) y .
$$

The assumption $x \neq 0$ implies that $y \neq 0$. Therefore,

$$
y=2(\beta B+\gamma A)^{-1} x,
$$

where $\beta=y^{\mathrm{T}} A y>0$ and $\gamma=y^{\mathrm{T}} B y>0$. Substituting (2) into $y^{\mathrm{T}} A y$ and $y^{\mathrm{T}} B y$, we have

$$
\begin{aligned}
& \beta=4 x^{\mathrm{T}}(\beta B+\gamma A)^{-1} A(\beta B+\gamma A)^{-1} x, \\
& \gamma=4 x^{\mathrm{T}}(\beta B+\gamma A)^{-1} B(\beta B+\gamma A)^{-1} x .
\end{aligned}
$$

Dividing (3) by (4) yields

$$
\frac{\beta}{\gamma}-\frac{x^{\mathrm{T}}\left(A+\frac{\beta}{\gamma} B\right)^{-1} A\left(A+\frac{\beta}{\gamma} B\right)^{-1} x}{x^{\mathrm{T}}\left(A+\frac{\beta}{\gamma} B\right)^{-1} B\left(A+\frac{\beta}{\gamma} B\right)^{-1} x}=0 .
$$

That is, $\alpha:=\frac{\beta}{\gamma}>0$ is a real root of (1). According to (3) and (4), we have

$$
\begin{aligned}
x^{\mathrm{T}} & (A+\alpha B)^{-1} x \\
& =x^{\mathrm{T}}(A+\alpha B)^{-1} A(A+\alpha B)^{-1} x+\alpha x^{\mathrm{T}}(A+\alpha B)^{-1} B(A+\alpha B)^{-1} x \\
& =\frac{1}{4} \beta \gamma^{2}+\frac{1}{4} \alpha \gamma^{3} \\
& =\frac{1}{2} \alpha \gamma^{3},
\end{aligned}
$$

which implies that

$$
\gamma=\left(\frac{2 x^{\mathrm{T}}(A+\alpha B)^{-1} x}{\alpha}\right)^{1 / 3} .
$$

Therefore, it follows from (2), (5), and the definitions of $\beta, \gamma$ and $\alpha$ that

$$
x^{\mathrm{T}} y-f(y)=\frac{2}{\gamma} x^{\mathrm{T}}(A+\alpha B)^{-1} x-\frac{1}{4} \beta \gamma=3 \alpha^{1 / 3}\left(\frac{x^{\mathrm{T}}(A+\alpha B)^{-1} x}{4}\right)^{2 / 3} .
$$

On the other hand, let $\alpha$ be a real root of Eq. (1). Then $(A+\alpha B)^{-1}$ is well defined and hence nonsingular. Since $A, B$ are positive definite, $(A+\alpha B)^{-1} A(A+\alpha B)^{-1}$ 
and $(A+\alpha B)^{-1} B(A+\alpha B)^{-1}$ are both positive definite. It follows from $x \neq 0$ that $x^{\mathrm{T}}(A+\alpha B)^{-1} A(A+\alpha B)^{-1} x>0$ and $x^{\mathrm{T}}(A+\alpha B)^{-1} B(A+\alpha B)^{-1} x>0$. Therefore, the formulation (1) implies $\alpha>0$. Now we can define $\gamma$ as in (5). Let $\beta=\alpha \gamma$. Define $y$ as in (2). Then $y$ is a stationary point. The proof is complete.

Now we present our main result.

Theorem 2.1 At any point $x \in \mathbb{R}^{n}$, the value of the conjugate $f^{*}(x)$ is finite, and $f^{*}(x)=0$ if $x=0$, otherwise if $x \neq 0$,

$$
f^{*}(x)=p\left(\alpha^{*}\right),
$$

$\alpha^{*}$ is a global maximizer of $p(\alpha)$.

Proof Following Lemma 2.1, it is sufficient to assume $x \neq 0$. Since the conjugate $f^{*}(x)$ is finite, $f^{*}(x)$ is attained at the global maximizer of $x^{\mathrm{T}} y-f(y)$, denoted by $y^{*}$. According to the necessary condition for unconstrained optimization, $y^{*}$ is a stationary point of $x^{\mathrm{T}} y-f(y)$. It follows from Lemma 2.2 that $f^{*}(x)=p\left(\alpha^{*}\right)$, where

$$
\alpha^{*}=\underset{g(\alpha)=0}{\operatorname{argmax}} p(\alpha) .
$$

It is not difficult to verify that the equations $\frac{d}{d \alpha} p(\alpha)=0$ and $g(\alpha)=0$ are equivalent. Therefore,

$$
\alpha^{*}=\operatorname{argmax} p(\alpha)
$$

which completes the proof.

Remark 1 The global maximizer of $p(\alpha)$ can be obtained by enumerating all the real roots of the univariate polynomial $g(\alpha)$, which is one of the best studied fundamental problems in computational algebra. It is sufficient to compute a set of disjoint isolating intervals, each containing exactly one root. There are numerous subdivision approaches such as the Descartes method [1, 4] and the Sturm Sequences [2]. When the polynomial has integer coefficients of maximal bitsize $\tau$, Sagraloff [4] recently proposed an algorithm with the complexity $\widetilde{O}\left(n^{3} \tau\right)$, where $n$ is the degree of the polynomial and $\widetilde{O}$ indicates that the logarithmic factors are omitted.

As an application of Theorem 2.1, we show that Theorem 1.2 can be improved.

Lemma 2.3 Suppose $x \neq 0 . x^{\mathrm{T}} y-f(y)$ has at most $n-1$ stationary points.

Proof According to Lemma 2.2, the number of stationary points of $x^{\mathrm{T}} y-f(y)$ is equal to the number of the real roots of Eq. (1). Since $A, B$ are positive definite, there exists a nonsingular matrix $P$ such that both $P^{\mathrm{T}} A P$ and $P^{\mathrm{T}} B P$ are diagonal matrices. We denote $P^{\mathrm{T}} A P=\operatorname{Diag}\left(a_{1}, \cdots, a_{n}\right)$ and $P^{\mathrm{T}} B P=\operatorname{Diag}\left(b_{1}, \cdots, b_{n}\right)$. Then, 
$a_{i}>0$ and $b_{i}>0$ for all $i=1, \cdots, n$. Let $z=P^{\mathrm{T}} x$. Clearly, $z \neq 0$, since $P$ is nonsingular and $x \neq 0$. Then we have

$$
\begin{aligned}
x^{\mathrm{T}} & (A+\alpha B)^{-1} A(A+\alpha B)^{-1} x \\
& =x^{\mathrm{T}} P\left(P^{\mathrm{T}}(A+\alpha B) P\right)^{-1} P^{\mathrm{T}} A P\left(P^{\mathrm{T}}(A+\alpha B) P\right)^{-1} P^{\mathrm{T}} x \\
& =z^{\mathrm{T}} \operatorname{Diag}\left(\frac{a_{1}}{\left(a_{1}+\alpha b_{1}\right)^{2}}, \cdots, \frac{a_{n}}{\left(a_{n}+\alpha b_{n}\right)^{2}}\right) z \\
& =\sum_{i=1}^{n} \frac{a_{i} z_{i}^{2}}{\left(a_{i}+\alpha b_{i}\right)^{2}}>0 .
\end{aligned}
$$

Similarly,

$$
x^{\mathrm{T}}(A+\alpha B)^{-1} B(A+\alpha B)^{-1} x=\sum_{i=1}^{n} \frac{b_{i} z_{i}^{2}}{\left(a_{i}+\alpha b_{i}\right)^{2}}>0 .
$$

Consequently, Eq. (1) is equivalent to

$$
\alpha \sum_{i=1}^{n} \frac{b_{i} z_{i}^{2}}{\left(a_{i}+\alpha b_{i}\right)^{2}}-\sum_{i=1}^{n} \frac{a_{i} z_{i}^{2}}{\left(a_{i}+\alpha b_{i}\right)^{2}}=0,
$$

which satisfies the following univariate polynomial equation:

$$
q(\alpha):=\left(\prod_{i=1}^{n}\left(a_{i}+\alpha b_{i}\right)^{2}\right)\left(\alpha \sum_{i=1}^{n} \frac{b_{i} z_{i}^{2}}{\left(a_{i}+\alpha b_{i}\right)^{2}}-\sum_{i=1}^{n} \frac{a_{i} z_{i}^{2}}{\left(a_{i}+\alpha b_{i}\right)^{2}}\right)=0 .
$$

One can verify that the leading coefficient of the polynomial $q(\alpha)$ is

$$
\left(\prod_{j=1}^{n} b_{j}^{2}\right)\left(\sum_{i=1}^{n} \frac{z_{i}^{2}}{b_{i}}\right) \alpha^{2 n-1}
$$

It follows from $b_{i}>0(i=1, \cdots, n)$ and $z \neq 0$ that (6) is a univariate polynomial equation of degree $(2 n-1)$. Therefore, Eq. (1) has at most $2 n-1$ real roots. The proof is complete.

Theorem 2.2 If $f(y)$ is convex, then for any $x \neq 0$, there exists a unique real root to Eq. (1).

Proof If $f(y)$ is convex, a stationary point of $x^{\mathrm{T}} y-f(y)$ is also a maximizer. It follows from Lemma 2.2 and Theorem 2.1 that any stationary point of $p(\alpha)$ is also a global maximizer of $p(\alpha)$. According to Lemma 2.3 and due to the equivalence of the equations $\frac{d}{d \alpha} p(\alpha)=0$ and $g(\alpha)=0, p(\alpha)$ has at most $2 n-1$ stationary points, denoted by $\alpha_{1}<\cdots<\alpha_{k}$, where $k \leqslant 2 n-1$. It is sufficient to show $k=1$. Suppose 
this is not true, i.e., $k \geqslant 2$. Since $p\left(\alpha_{1}\right)=p\left(\alpha_{2}\right)=\max _{\alpha>0} p(\alpha)$ and $\frac{d}{d \alpha} p(\alpha) \neq 0$ for any $\alpha \in\left(\alpha_{1}, \alpha_{2}\right)$, we have $p(\alpha)<p\left(\alpha_{1}\right)$ for all $\alpha \in\left(\alpha_{1}, \alpha_{2}\right)$. Define

$$
\widetilde{\alpha}=\underset{\alpha \in\left(\alpha_{1}, \alpha_{2}\right)}{\operatorname{argmin}} p(\alpha)
$$

Then $\widetilde{\alpha}$ is a stationary point of $p(\alpha)$ and cannot be a maximizer of $p(\alpha)$. This contradiction completes the proof.

\section{Conclusion}

The Legendre-Fenchel conjugate of the product of two positive-definite quadratic forms was posted as an open question by Hiriart-Urruty [3]. Under a convexity assumption on the function, it was answered by Zhao [5]. In this note, we give an answer to the open question without making the convexity assumption. When restricted to the convex case, our new proof is much shorter than that of [5]. Moreover, we improve a sufficient condition given in [5] on the uniqueness of the root of the univariate equation for deriving the conjugate. Finally, we raise a question whether our approach can be extended to the Legendre-Fenchel conjugate for the product of finitely many positive definite quadratic forms [6].

Acknowledgements The author is grateful to the two anonymous referees whose comments improved this paper. The author also thanks Prof. Yun-Bin Zhao, Prof. Henry Wolkowicz and his doctoral student Minghua Lin for valuable comments.

\section{References}

[1] Collins, G.E., Akritas, A.G.: Polynomial real root isolation using Descartes' rule of signs. In: Proc. of the 3rd ACM Symp. on Symbolic and Algebraic Computation (SYMSAC 1976), pp. 272-275. ACM Press, New York (1976)

[2] Du, Z., Sharma, V., Yap, C.: Amortized bound for root isolation via Sturm sequences. In: SymbolicNumeric Computation. Trends in Mathematics, pp. 113-129. Birkhäuser, Basel (2007)

[3] Hiriart-Urruty, J.B.: Potpourri of conjectures and open questions in nonlinear analysis and optimization. SIAM Rev. 49, 255-273 (2007)

[4] Sagraloff, M.: When Newton meets Descartes: a simple and fast algorithm to isolate the real roots of a polynomial. In: Proceedings of the 37th International Symposium on Symbolic and Algebraic Computation, pp. 297-304. ACM Press, New York (2012)

[5] Zhao, Y.B.: The Legendre-Fenchel conjugate of the product of two positive-definite quadratic forms. SIAM J. Matrix Anal. Appl. 31(4), 1792-1811 (2010)

[6] Zhao, Y.B.: Convexity conditions and the Legendre-Fenchel transform for the product of finitely many positive definite quadratic forms. Appl. Math. Optim. 62(3), 411-434 (2010) 